\title{
Relevance of Lifestyle and Attitudinal Factors to Spine Surgery Outcomes: Empirical Results on a Heterogeneous Sample
}

\author{
Carolyn E. Schwartz ${ }^{1,2}$, Brian Quaranto ${ }^{1,3,4}$, Emily Samaha ${ }^{3,4}$, Mariam Kahn-Woods ${ }^{4,5}$, Paul Glazer ${ }^{6}$ \\ ${ }^{1}$ DeltaQuest Foundation, Concord, MA, USA \\ ${ }^{2}$ Departments of Medicine and Orthopaedic Surgery, Tufts University School of Medicine, Boston, MA, USA \\ ${ }^{3}$ Boston College, Chestnut Hill, MA, USA \\ ${ }^{4}$ Foundation for Orthopaedic Spine Research, Chestnut Hill, MA, USA \\ ${ }^{5}$ University of Pennsylvania School of Nursing, Philadelphia, PA, USA \\ ${ }^{6}$ Department of Orthopaedic Surgery, Beth Israel Deaconess Medical Center, \\ Harvard Medical School, Boston, MA, USA \\ E-mail: carolyn.schwartz@deltaquest.org \\ Received January 13, 2011; revised April 4, 2011; accepted April 12, 2011
}

\begin{abstract}
Background Context: Patient demographic and medical indicators influence the well-being of spine surgery patients. It may, however, be worthwhile to evaluate other lifestyle and attitudinal factors. We hypothesized that such factors would explain at least as much variance in outcome as more commonly considered covariates. Purpose: To compare explained variance in outcome of lifestyle and attitudinal factors as compared to standard demographic and medical covariates. Study Design/Setting: Cross-sectional observational study of patients drawn from an active clinic and internet-based support group. Patient Sample: A heterogeneous sample of 376 patients was recruited, comprised of people with diagnoses of cervical $(n=80)$, lumbar $(n=$ 228), and scoliosis $(\mathrm{n}=68)$ spine disorders. Outcome Measures: Quality of Life (QOL) outcomes were measured using the Oswestry Disability Index, Neck Disability Index, Rand-36, PROMIS Pain Impact, NRS Back and Leg Pain, Scoliosis Research Society-22r, and Global Health. Methods: This study compared explained variance in QOL outcomes of demographic and medical versus lifestyle and attitudinal factors. Demographic and medical factors included age, gender, body mass index, and co-morbidities. Lifestyle factors included exercise and commuting practice. Attitudinal factors related to social connectedness: giving and receiving emotional support, feeling overwhelmed by others' needs, helping orientation, and general helping behaviors. Regression analyses estimated explained variance. Patient groups differed in most factors evaluated, so the regression analyses were computed separately by group. R2 statistics were characterized as null, small (0.02), medium (0.15), and large (0.35) effect sizes (ES), and proportions were compared for the medical/demographic versus lifestyle/attitudinal factors by group. Results: Similar proportions of variance were explained by demographic/medical and lifestyle/attitudinal covariates across groups, with half of effect sizes being small in magnitude and $6 \%$ being medium. Lumbar patients tended to have more small effect sizes among lifestyle and attitudinal covariates than among medical/demographic covariates $(\mathrm{z}=-1.29, \mathrm{p}<0.10)$. Similar patterns were found for both generic and disease-specific outcomes. Conclusions: Spine surgery outcome research should investigate lifestyle and attitudinal factors to enhance the personal and salutogenic relevance of the research. Time spent commuting, exercise practice, and social connectedness appear to be relevant factors. A pre-operative evaluation of overweight and smoking status, limited social connectedness, and long daily commutes could alert the surgeon to delay or avoid performing procedures on these patients to avoid poor outcomes.
\end{abstract}

Keywords: Spine Surgery Outcomes, Predictors, Behaviors, Attitudes, Lumbar, Cervical, Scoliosis 


\section{Background Context}

Medical outcome research has evolved greatly in recent years, with increasing sophistication in estimating effects of medical and surgical interventions to quantify the importance of such effects. Measurement science has extended such work to facilitate the interpretation of changes in outcomes in terms of their clinical significance [1-3], and has paved the way for evidence-based clinical treatment guidelines [4-6]. As part of this evolution, studies in spine research have documented a wide range of factors that influence treatment outcome, including medical factors (e.g., co-morbidities [7]), sociodemographic factors [8] (e.g., gender, age), and behavioral factors $[8,9]$ (workers' compensation status $[10,11]$ ).

A recent book on psychological factors related to spine surgery noted a cluster of about ten psychosocial and medical risk factors with sufficient empirical documentation to merit continued consideration [8]. Such factors included psychological or personality factors (e.g., hostility, anxiety, depression, history of psychological disturbance), behavioral factors (e.g., smoking, substance abuse, worker's compensation status, obesity), social support, and attitudinal factors (e.g., job dissatisfyaction) [8]. They note that psychosocial factors are often found to be stronger predictors of surgical outcome than are medical diagnostic factors, and derived an algorithm for determining surgical prognosis on the basis of these risk factors [8]. We believe that more research on psychosocial factors in spine outcome research is warranted, and present preliminary findings to support this recommendation.

\section{Purpose}

The purpose of the present work was to evaluate the association of several heretofore-unexamined lifestyle and attitudinal factors with quality-of-life (QOL) outcomes in a sample of patients with spinal disorders, and to compare variance explained by these factors and more commonly considered medical and demographic factors.

\section{Methods}

\subsection{Design}

To achieve a heterogeneous sample, this cross-sectional study recruited patients from different sources. First, patients were recruited from an active spine surgeon's practice, comprised of people with diagnoses of cervical, lumbar, and scoliosis spine disorders. Over half of the scoliosis patient sample (53\%) was additionally recruited from other sources, including local support groups, via an article in the Scoliosis Association of America, and via an internet chat room for people with flatback syndrome. Second, this study included people representing a broad range of time since surgery and number of spinal surgeries. Approximately one quarter of the data were collected within a month of a planned spinal surgery, and three quarters of the sample had an average of 4.1 years since surgery. Since many patients have had repeated spinal surgeries over the course of their lifetime, combining them into one analytic sample is a reasonable approach to enhancing the generalizability of the findings as well as maximizing the sample size. Thus, it is important to note that in the present work, "outcomes" refers to standardized measures of physical, psychological, and social functioning and well-being, which can pertain to spine surgery patients at all stages of the treatment trajectory.

\subsection{Patient Sample}

Three hundred and seventy-six people with diagnoses of cervical $(n=80)$, lumbar $(n=228)$, and scoliosis $(n=68)$ spine disorders.

\subsection{Outcome Measures}

Quality of Life (QOL) outcomes were measured using the self-reported Oswestry Disability Index (ODI) [12], Neck Disability Index (NDI) [12,13], Rand-36 [14] for cervical and lumbar patients and the Rand-12 [15] for scoliosis patients, PROMIS ${ }^{1}$ Pain Impact [16], Numeric Rating Scale (NRS) items for Back and Leg Pain [17], Scoliosis Research Society-22r [18], and a 10-point Likert scaled Global Health item. Co-morbidities were measured by the Self-Administered Co-morbidity Questionnaire [19]. Demographic factors included age in years, gender, dummy variables for normal- and obesebody mass index (BMI), having a college education, current smoker, currently on worker's compensation, a summative score of co-morbidities, and whether the patient endorsed having diabetes or depression. Lifestyle measures included: 1) a three-item index of exercise comprised of strength-building, aerobic activity, and yoga or Pilates, with response options of rarely/never (a), 1 - 2 times per week (b), or 3 or more times per week (c); and 2) commuting practices, as measured by daily amount of time in hours spent commuting. Attitudinal factors included the Schwartz Altruism Questionnaire, a validated 18 -item measure self-report measure that assesses four aspects of social connectedness: Community Connection, Community Pressure, Helping Orientation,

${ }^{1}$ Refers to measure developed by NIH Roadmap Initiative called the Patient-Reported Outcome Measurement Information System (PROMIS). This measure is a static short-form. See www.nihpromis.org for details. 
and General Helping Behavior [20].

\subsection{Procedure}

The study was reviewed and approved by the Beth Israel Deaconess Medical Center Institutional Review Board, and all patients provided written informed consent prior to completing the questionnaires. Data were collected online using a secure, HIPAA-compliant interface (www.surveygizmo.com).

\subsection{Statistical Analysis}

This study compared explained variance in QOL outcomes in demographic and medical factors versus lifestyle and attitudinal factors. Linear regressions estimated explained variance. R2 statistics were characterized as null, small (0.02), medium (0.15); and large (0.35) effect sizes (ES) [21], and proportions were compared for the demographic/medical vs. lifestyle and attitudinal factors by group.

\section{Results}

Descriptive statistics for the three patient groups are presented in Table 1. The three patient groups differed in most factors evaluated, with the scoliosis patients being younger, a higher proportion female, a smaller proportion overweight or obese, and a larger proportion with some college education. Scoliosis patients also reported the longest time since surgery, with a mean of over 13 years as compared to a mean of about 2 years in the cervical and lumbar patients. Scoliosis patients also had a higher number of surgeries as compared to the cervical and lumbar patients (mean $=1.64,1.2$, and 1.1, respectively). The cervical and lumbar patients reported similar numbers of medical co-morbidities, and substantially fewer co-morbidities than the scoliosis patients. The prevalence of depression and diabetes was highest among cervical patients.

Regarding the lifestyle factors investigated, the scoliosis patients reported more frequent engagement in all types of exercise, with a particularly notable difference in the practice of yoga or Pilates compared to the other patient groups. Similar proportions of patients were of Caucasian race, married, currently employed, and current smokers. The mean time spent commuting was also similar across groups. Regarding the attitudinal factors, patient groups differed on four of the five subscales of the Altruism scale, with scoliosis patients reporting lower levels of giving support and helping orientations, higher reported levels of feeling overwhelmed by others' demands, and higher reported levels of engaging in general helping behaviors. There were no differences in reported
Table 1. Descriptive statistics of subsamples.

\begin{tabular}{|c|c|c|c|c|}
\hline & $\begin{array}{l}\text { Cervical } \\
(n=80)\end{array}$ & $\begin{array}{l}\text { Lumbar } \\
(n=228)\end{array}$ & $\begin{array}{l}\text { Scoliosis } \\
(n=68)\end{array}$ & $\begin{array}{l}F \text { or } X^{2} \\
\text { Statistic }^{a}\end{array}$ \\
\hline \multicolumn{5}{|c|}{ Demographic and Medical Characteristics } \\
\hline Mean Age (Sd) & $57.9(13.6)$ & $56.9(14.3)$ & $54.7(11.9)$ & 0.55 \\
\hline Gender: \% Female & 58.4 & 48.0 & 92.6 & $38.7 * * * *$ \\
\hline $\begin{array}{l}\% \text { Adolescent Onset } \\
\text { Scoliosis }\end{array}$ & - & - & 69.1 & - \\
\hline $\begin{array}{l}\text { Mean Body Mass } \\
\text { Index (Sd) }\end{array}$ & $27.5(7.2)$ & $27.8(5.3)$ & $24.7(3.6)$ & $10.85^{* * *}$ \\
\hline$\%$ Underweight & 10.0 & 6.3 & 0 & \multirow{4}{*}{$26.5 * * * *$} \\
\hline$\%$ Normal Weight & 27.1 & 28.3 & 58.6 & \\
\hline$\%$ Overweight & 37.1 & 40.3 & 34.5 & \\
\hline$\%$ Obese & 25.7 & 25.1 & 6.9 & \\
\hline Marital: \% Married & 64.5 & 64.0 & 65.1 & 0.03 \\
\hline Race: \% Caucasian & 94.9 & 92.8 & 95.0 & 4.07 \\
\hline $\begin{array}{l}\text { Education: \% Some } \\
\text { College or Greater }\end{array}$ & 80.0 & 82.4 & 92.6 & 7.42 \\
\hline \% Currently Working & 45.1 & 42.9 & 43.9 & 18.6 \\
\hline $\begin{array}{l}\% \text { Worker'S Com- } \\
\text { pensation }\end{array}$ & 0 & 0.44 & 0 & $11.2^{*}$ \\
\hline $\begin{array}{l}\text { Smoking: \% Current } \\
\text { Smokers }\end{array}$ & 8.75 & 7.46 & - & $11.9 \dagger$ \\
\hline $\begin{array}{l}\text { Katz Comorbidity } \\
\text { Score Mean (Sd) }\end{array}$ & $3.91(3.69)$ & $3.28(3.50)$ & $5.00(3.75)$ & $11.1 * * *$ \\
\hline $\begin{array}{l}\% \text { Depression } \\
\text { Comorbidity }\end{array}$ & 32.1 & 22.9 & 20.3 & 3.9 \\
\hline
\end{tabular}

\section{Lifestyle Characteristics}

Strength-building (sd) $1.67(0.85) \quad 1.80(0.84) \quad 2.03(0.83)$

$\begin{array}{lllll}\text { Aerobic (sd) } \quad 1.72(0.91) & 1.79(0.89) & 2.01(0.83) & 15.8^{* *}\end{array}$

Yoga/Pilates (sd) $\quad 1.03(0.23) \quad 1.11(0.73) \quad 2.59(0.74) \quad 233.7^{* * *}$

Total (sd) $\quad 4.39(1.59) \quad 4.68(1.63) \quad 6.63(1.64) \quad 34.9^{* * * *}$

Mean Travel $\quad 1.40(1.96) \quad 1.31(1.01) \quad 1.54(1.23) \quad 1.29$

hours/day (sd)

Attitudinal Characteristics

Altruism Scale

$\begin{array}{lllll}\text { Receive Support (sd) } & 1.87(0.84) & 1.96(0.84) & 2.07(.67) & 0.09\end{array}$

$\begin{array}{lllll}\text { Give Support (sd) } \quad 2.09(.80) & 2.01(.91) & 1.84(0.70) & 0.66\end{array}$

Overwhelm (sd) $\quad 0.78(0.82) \quad 0.82(0.80) \quad 2.42(1.1) \quad 90.02 * * * *$

$\begin{aligned} & \text { Helping Orientation } \\ & (\mathrm{sd})\end{aligned}$
$4.64(0.71)$$\quad 4.31(1.1) \quad 1.88(1.2) \quad 102.76^{* * * *}$

$\begin{array}{llll}\text { General Helping } & 2.04(0.81) & 1.91(0.92) & 2.81(1.0) \\ \text { Behaviors (sd) } & 36.00^{* * * *}\end{array}$

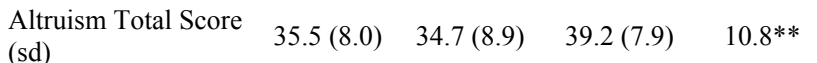

a. $\dagger \mathrm{p}<0.10, * \mathrm{p}<0.05, * * \mathrm{p}<0.01, * * * \mathrm{p}<0.001, * * * * \mathrm{p}<0.0001$ 
Table 2. Outcome scores of the subsamples.

\begin{tabular}{|c|c|c|c|c|}
\hline & $\begin{array}{l}\text { Cervical } \\
(n=80)\end{array}$ & $\begin{array}{c}\text { Lumbar } \\
(n=228)\end{array}$ & $\begin{array}{l}\text { Scoliosis } \\
(n=68)\end{array}$ & $\begin{array}{l}\text { F or } X^{2} \\
\text { Statistic }\end{array}$ \\
\hline $\begin{array}{l}\text { Mean SF-36 Physi- } \\
\text { cal Component } \\
\text { Score (sd) }\end{array}$ & $\begin{array}{c}40.1 \\
(11.2)\end{array}$ & $\begin{array}{c}39.7 \\
(11.9)\end{array}$ & - & 0.32 \\
\hline $\begin{array}{l}\text { Mean SF-36 Mental } \\
\text { Component Score } \\
\text { (sd) }\end{array}$ & $\begin{array}{c}45.2 \\
(14.2)\end{array}$ & $\begin{array}{c}46.1 \\
(13.1)\end{array}$ & - & 1.28 \\
\hline $\begin{array}{l}\text { Mean SF-12 Physi- } \\
\text { cal Component } \\
\text { Score (sd) }\end{array}$ & - & - & $\begin{array}{c}40.1 \\
(11.2)\end{array}$ & - \\
\hline $\begin{array}{l}\text { Mean SF-12 Mental } \\
\text { Component Score } \\
\text { (sd) }\end{array}$ & - & - & $\begin{array}{c}49.1 \\
(11.7)\end{array}$ & - \\
\hline $\begin{array}{l}\text { Mean Scoliosis } \\
\text { Research Soci- } \\
\text { ety-22r Total Score } \\
\text { (sd) }\end{array}$ & - & - & $\begin{array}{l}3.5 \\
(0.8)\end{array}$ & - \\
\hline $\begin{array}{l}\text { Oswestry Disability } \\
\text { Index (sd) }\end{array}$ & - & $\begin{array}{c}42.3 \\
(16.8)\end{array}$ & --- & 0.41 \\
\hline $\begin{array}{l}\text { Neck Disability } \\
\text { Index (sd) }\end{array}$ & $\begin{array}{c}53.7 \\
(19.0)\end{array}$ & - & - & \\
\hline NRS Leg Pain (sd) & $\begin{array}{l}2.2 \\
(2.6)\end{array}$ & $\begin{array}{c}3.2 \\
(3.06)\end{array}$ & - & $6.99 * *$ \\
\hline NRS Back Pain (sd) & $\begin{array}{c}3.0 \\
(2.93)\end{array}$ & $\begin{array}{c}3.8 \\
(2.95)\end{array}$ & - & $3.84 *$ \\
\hline $\begin{array}{l}\text { PROMIS Pain } \\
\text { Impact Short-Form } \\
\text { T score (sd) }\end{array}$ & $\begin{array}{l}58.6 \\
(10.5)\end{array}$ & $\begin{array}{c}59.4 \\
(10.3)\end{array}$ & - & 0.54 \\
\hline Global Health (sd) & $\begin{array}{c}6.5 \\
(2.1)\end{array}$ & $\begin{array}{c}6.4 \\
(2.1)\end{array}$ & $\begin{array}{c}6.8 \\
(2.0)\end{array}$ & 1.82 \\
\hline
\end{tabular}

levels of received support.

Table 2 presents mean outcome scores by patient group. There were similar scores on the RAND physical and mental component scores, the ODI/NDI, the PROMIS Pain Impact Score, and the Global Health item. Patient responses to the NRS leg and back pain items differed, with lumbar patients reporting higher levels of both leg and back pain as compared to cervical patients.

Figure 1 summarizes the findings from the univariate regressions examining explained variance in QOL outcome by type of covariate. We found that adjusting for whether the data were drawn from the pre-surgical vs. post-surgical sample did not change the effect size for the covariates of interest. Thus, the reported regression models did not adjust for type of sample. Similar proportions of variance were explained by demographic/medical and behavioral covariates across groups, with half of effect sizes being small in magnitude and $6 \%$ being medium. Lumbar patients tended to have more small effect sizes among behavioral covariates (51\%) than among medical/demographic covariates $(37 \%)(\mathrm{z}=-$ $1.29, \mathrm{p}<0.10)$. Similar patterns were found for both generic and disease-specific outcomes.

Figure 2 shows more detail in explained variance in

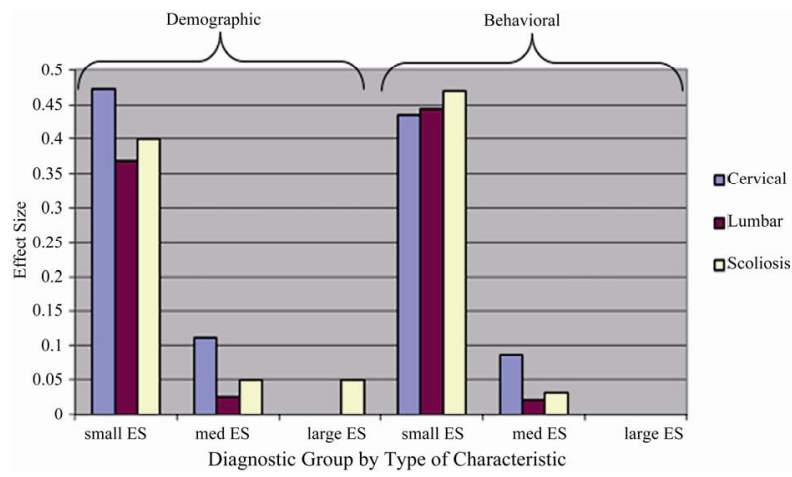

Figure 1. A comparison of variance explained by demographic versus behavioral characteristics. Summarizes the findings from the univariate regressions examining variance in QOL outcomes by type of covariate. Similar proportions of variance were explained by demographic/ medical and behavioral (lifestyle/attitudinal) covariates across groups.

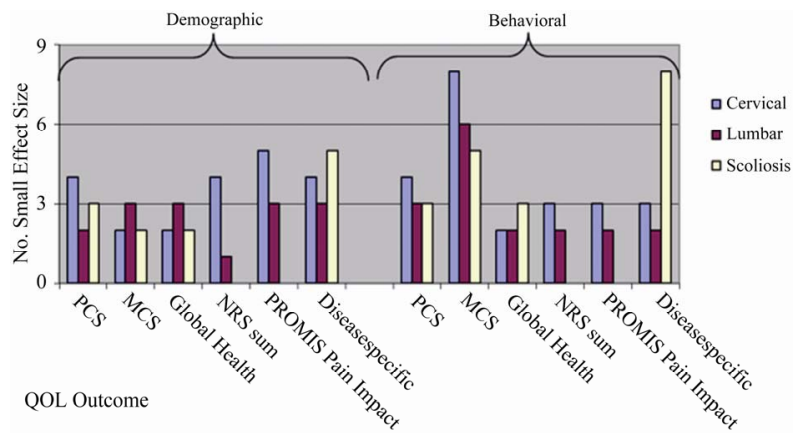

Figure 2. Number of small effect sizes by QOL outcome, separately by diagnostic group. Key points are that generic and disease-specific outcomes appeared to yield similar numbers of statistically important effect sizes, although there were more statistically important behavioral covariates predicting MCS scores than any other QOL outcome. Additionally, cervical patients generally had more effect sizes across the QOL outcomes than other patient groups.
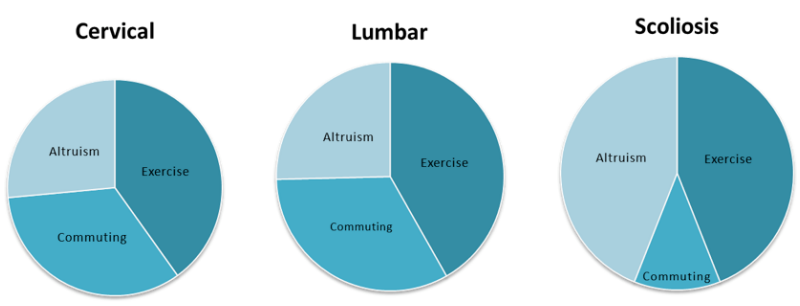

Figure 3. Relative importance of behavioral covariates to QOL outcomes. All three types of behavioral covariates are important across patient groups and, for cervical and lumbar patients, have similar relative importance for the cervical and lumbar patients. For scoliosis patients, however, altruism and exercise are more important than commuting practice for explaining spine outcomes.

QOL outcomes by groups. For the sake of simplicity, the number of small effect sizes is plotted, since these were 
the most prevalent effect sizes detected. This figure highlights several points. First, cervical patients generally had more effect sizes across the QOL outcomes than other patient groups. Second, there appeared to be a larger number of statistically important behavioral covariates predicting MCS scores than any other QOL outcome. Third, generic and disease-specific outcomes appeared to yield similar numbers of statistically important effect sizes, with the above-noted exception of MCS. The disease-specific scoliosis measure appeared to have substantially more statistically important effect sizes among behavioral covariates, suggesting that these factors are particularly important for this patient population.

Figure 3 shows the proportion of QOL outcome measures for which each behavioral characteristic is statistically important. These pie charts suggest that all three types of behavioral covariates are important across patient groups. Additionally, the three types of behavioral covariates have similar relative importance for the cervical and lumbar patients, but for scoliosis patients, altruism and exercise are more important than commuting practice for explaining spine outcomes.

\section{Discussion}

This study suggests that in addition to considering known demographic and medical factors related to patient outcome, spine surgery researchers should also consider including measures of lifestyle and behavioral factors. Factors such as exercise practice, average daily commute, and social connectedness were found to have similar value in explaining condition-specific and generic outcome measures as those demographic and medical covariates generally considered in spine outcome research. In our heterogeneous spine patient sample, the effect sizes were generally small for the examined covariates. Nonetheless, such factors may have a synergistic effect on treatment outcomes, such that adjusting for them increases the detected effect of clinical interventions.

This study utilized a heterogeneous sample of people with spinal disorders expressly as a first, exploratory endeavor to evaluate the utility of such lifestyle and attitudinal factors. To build on this preliminary work, future research might evaluate the relationship between these factors and patient outcomes, after adjusting for the demographic and medical factors. It would be useful to know, for example, whether the examined aspects of exercise practice, commuting burden, and social connectedness have a similar association with outcomes across patient age, gender, co-morbidity, diagnosis, and surgery subgroups. Engaging in a balanced regimen of exercise (i.e., integrating aerobic, strengthening, and stretching types of exercise) may reflect other important patient factors, including socioeconomic status, amount of leisure time, education level, and motivation. Commuting burden may also reflect other factors, such as type of occupation (i.e., more or less amenable to telecommuting, professional occupation rather than laborer or service occupation), education level, and income status (i.e., longer commutes are often related to lower rent/housing cost further from the urban center). Social connectedness may play a more important role later in the recovery process, and may be more relevant for mental health outcomes rather than disability outcomes. In past research in adults, such altruistic social interest behaviors were associated with higher levels of mental health [22], and lower mortality [23], and males and females seemed to have different aspects of well-being associated with such behaviors [20].

The limitations of the present work include the relatively small numbers of cervical and scoliosis patients that prevent subgroup analyses, and the cross-sectional design that prevents causal inference. These limitations are offset by the strength lent by the heterogeneous recruitment strategy. This strategy enhanced the generalizability and statistical power to detect small effect sizes.

Future research using the analytical approach exemplified above may predict poorer outcomes in specific patient population subsets (such as those who are overweight, smoke, have limited social connectedness, and have longer commutes). A pre-operative evaluation of these factors might then alert the surgeon to delay or avoid performing procedures on these patients to avoid poor outcomes.

In conclusion, this study supports the importance of including lifestyle and attitudinal factors in an evaluation of spine surgery outcomes. Such factors should be considered relevant as covariates for adjusting in data analysis, and possibly even as stratification or matching in clinical trials. Further, such characteristics should be addressed clinically before and after spine surgery, since our findings suggest that they are relevant to QOL and well-being. One might, for example, encourage the balanced integration of exercise as soon as it is safe after surgery to improve patient outcomes, both directly and via reduced BMI. Encouraging the patient to consider ways to reduce daily time spent in a car may also result in better outcomes, both by reducing back pain symptoms and by freeing up time in the day for activities that enhance fitness (i.e., exercise) and mood (e.g., social connectedness activities).

\section{Acknowledgements}

Funding for this work was provided in part from the 
Foundation for Orthopaedic Spine Research.

\section{References}

[1] J. A. Sloan, D. Cella and R. D. Hays, "Clinical SigniFicance of Patient-Reported Questionnaire Data: Another Step toward Consensus. (Editorial)," Journal Clinical Epidemiology, Vol. 58, No. 12, 2005, pp. 1217-1219. doi:10.1016/j.jclinepi.2005.07.009

[2] L. Y. Carreon, et al., "Neck Disability Index, Short Form-36 Physical Component Summary, and Pain Scales for Neck and Arm Pain: The Minimum Clinically Important Difference and Substantial Clinical Benefit after Cervical Spine Fusion," The Spine Journal, Vol. 10, No. 6, 2010, pp. 469-474. doi:10.1016/j.spinee.2010.02.007

[3] B. R. Theodore, "Methodological Problems Associated with the Present Conceptualization of the Minimum Clinically Important Difference and Substantial Clinical Benefit," The Spine Journal, Vol. 10, No. 6, 2010, pp. 507-509. doi:10.1016/j.spinee.2010.04.003

[4] D. K. Resnick, "Evidence-Based Spine Surgery," Spine, Vol. 32, Supplement 11, 2007, pp. S15-19. doi:10.1097/BRS.0b013e318053d4b4

[5] W. C. Watters, et al., "Degenerative Lumbar Spinal Stenosis: An Evidence-Based Clinical Guideline for the Diagnosis and Treatment of Degenerative Lumbar Spinal Stenosis," The Spine Journal, Vol. 8, No. 2, 2008, pp. 305-310.

[6] W. C. Watters, et al., "An Evidence-Based Clinical Guide-Line for the Diagnosis and Treatment of Degenerative Lumbar Spondylolisthesis," The Spine Journal, Vol. 9, No. 7, 2009, pp. 609-614. doi:10.1016/j.spinee.2009.03.016

[7] J. Slover, et al., "The Impact of Comorbidities on the Change in Short-Form 36 and Oswestry Scores Following Lumbar Spine Surgery," Spine, Vol. 31, No. 7, 2006, pp. 1974-1980. doi:10.1097/01.brs.0000229252.30903.b9

[8] A. R. Block, et al., "The Psychology of Spine Surgery," American Psychological Association, Washington DC, 2003. doi:10.1037/10613-000

[9] B. Van Dorsten and E. M. Lindley, "Improving Outcomes via Behavioral Assessment of Spine Surgery Candidates," SpineLine, January/February 2010, pp. 15-20.

[10] I. Harris, et al., "Association between Compensation Stat-Us and Outcome after Surgery: A Meta-Analysis," Journal of the American Medical Association, Vol. 293, No. 13,2005 , pp. 1644-1652. doi:10.1001/jama.293.13.1644

[11] M. L. Rohling, L. M. Binder and J. LanghinrichsenRohling, "Money Matters: A Meta-Analytic Review of the Association between Financial Compensation and the
Experience and Treatment of Chronic Pain," Health Psychology, Vol. 14, No. 6, 1995, pp. 537-547. doi:10.1037/0278-6133.14.6.537

[12] J. C. Fairbank and P. B. Pynsent, "The Oswestry Disability Index," Spine, Vol. 25, No. 22, 2000, pp. 2940-2952. doi:10.1097/00007632-200011150-00017

[13] H. Vernon and S. Mior, "The Neck Disability Index: A Study of Reliability and Validity," Journal of Manipulative Physiological Therapy, Vol. 14, 1991, pp. 405-415.

[14] R. D. Hays, C. D. Sherbourne and R. Maze, "The RAND 36-Item Health Survey 1.0," Health Economics, Vol. 2, No. 3, 1993, pp. 217-227. doi:10.1002/hec.4730020305

[15] J. Ware Jr., M. Kosinski and S. D. Keller, "A 12-Item Short-Form Health Survey: Construction of Scales and Preliminary Tests of Reliability and Validity," Medical Care, Vol. 34, No. 3, 1996, pp. 220-233. doi:10.1097/00005650-199603000-00003

[16] K. F. Cook, M. A. Kallen and D. Amtmann, "Having a Fit: Impact of Number of Items and Distribution of Data on Traditional Criteria for Assessing IRT's Unidimensionality Assumption," Quality of Life Research, Vol. 18, No. 4, 2009, pp. 447-460. doi:10.1007/s11136-009-9464-4

[17] R. A. Deyo, et al., "Outcome Measures for Low Back Pain Research. A Proposal for Standardized Use," Spine, Vol. 23, No. 18, 1998, pp. 2003-2013. doi:10.1097/00007632-199809150-00018

[18] K. H. Bridwell, et al., "Is the SRS-22 Instrument Responsive to Change in Adult Scoliosis Patients Having Primary Spinal Deformity Surgery?" Spine, Vol. 32, No. 20, 2007, pp. 2220-2225. doi:10.1097/BRS.0b013e31814cf120

[19] O. Sangha, et al., "The Self-Administered Comorbidity Questionnaire: A New Method to Assess Comorbidity for Clinical and Health Services Research," Arthritis Rheum, Vol. 49, No. 2, 2003, pp. 156-163. doi:10.1002/art.10993

[20] C. E. Schwartz, et al., "Helping Others Shows Differential Benefits on Health and Well-Being for Male and Female Teens," Journal of Happiness Studies, Vol. 10, No. 4, 2009, pp. 431-448. doi:10.1007/s10902-008-9098-1

[21] J. Cohen, "A Power Primer," Psychological Bulletin, Vol. 112, No. 1, 1992, pp. 155-159. doi:10.1037/0033-2909.112.1.155

[22] C. Schwartz, et al., "Altruistic Social Interest Behaviors Are Associated with Better Mental Health," Psychosomatic Medicine, Vol. 65, No. 5, 2003, pp. 778-785. doi:10.1097/01.PSY.0000079378.39062.D4

[23] S. L. Brown, et al., "Religion and Emotional CompenSation: Results from a Prospective Study of Widowhood," Personality and Social Psycholical Bulletin, Vol. 30, No. 9, 2004, pp. 1165-1174. doi: $10.1177 / 0146167204263752$ 